\title{
On Quasi-Sasakian Manifolds
}

\author{
Ahmet Sazak ${ }^{1}$ and Ahmet Yıldız ${ }^{2 *}$ \\ ${ }^{1}$ Department of Mathematics, Faculty of Science and Arts, Muş Alparslan University, Mus, Turkey \\ ${ }^{2}$ Department of Mathematics, Education Faculty, Inonu University, Malatya, Turkey \\ ${ }^{*}$ Corresponding authorE-mail: a.yildiz@inonu.edu.tr
}

\author{
Article Info \\ Keywords: Quasi-Sasakian manifolds, \\ Schouten-van Kampen connection, $\mathscr{D}_{\alpha^{-}}$ \\ homothetic deformation \\ 2010 AMS: 53C07, 53C25, 53D15 \\ Received: 15 February 2019 \\ Accepted: 22 March 2019 \\ Available online: 17 June 2019
}

\begin{abstract}
In this paper we study three-dimensional quasi-Sasakian manifolds admitting the Schoutenvan Kampen connection. Also, we study D-homothetic deformations on three-dimensional quasi-Sasakian manifolds admitting Schouten-van connection and projectively flat threedimensional quasi-Sasakian manifolds admitting scv connection.
\end{abstract}

\section{Introduction}

An important class of almost contact metric (shortly a.c.m.) manifolds is the class consisting of those which are normal. However, the curvature nature of such manifolds is not known in general, except for Sasakian or cosymplectic manifolds. If the almost contact structure (shortly a.c.s.) is normal and the fundamental 2 -form is closed then the manifold $M$ is called a quasi-Sasakian manifold (shortly q.S.).

First examples of q.S. manifolds were given by D. E. Blair [1]. Also, some remarks on q.S. structures given by S. Tanno [2]. Then, on a three-dimensional q.S. manifold the structure function $\gamma$ was introduced by Z. Olszak [3].

The Schouten-van Kampen connection (shortly S.K.con.) has been introduced of non-holomorphic manifolds. Then the S.K.con. was applied to a.c.m. structure by Z. Olszak and he characterized some classes of a.c.m manifolds [4]. Also, A. Yildiz studied three-dimensional $f$-Kenmotsu manifolds according to this connection [5].

In the present paper, we study three-dimensional q.S. manifolds with a $\mathscr{D}_{\alpha}$-homothetic deformation admitting the S.K.con..

\section{Preliminaries}

Let $\varphi$ is $(1,1)$-type tensor field, $\xi$ is a locally defined vector field tangent to $M$ and $\eta$ is a 1-form on $M$. Then $M(\varphi, \xi, \eta, g)$ is called an a.c.m. manifold whose elementary properties are [6]-[8]

$$
\begin{gathered}
\varphi^{2}=-I+\eta \otimes \xi, \quad \eta(\xi)=1, \\
g(\varphi U, \varphi V)=g(U, V)-\eta(U) \eta(V) . \\
\varphi \xi=0, \quad \eta \circ \varphi=0, \quad \eta(U)=g(U, \xi) .
\end{gathered}
$$

The fundamental 2-form $\theta$ is defined by

$$
\theta(U, V)=g(U, \varphi V) .
$$

Thus $\theta(U, \xi)=0$, for $U \in T M$. If the a.c.s. $(\varphi, \xi, \eta)$ is normal, i.e., $[\varphi, \varphi](U, U)+d \eta(U, V) \xi=0$ and the fundamental 2-form $\theta$ is closed, i.e. $d \theta=0$, then $M$ is called a q.S. manifold. An a.c.m. manifold $M$ is a three-dimensional q.S. manifold if and only if [9]

$$
\nabla_{U} \xi=-\gamma \varphi U
$$


for a function $\gamma$ on $M$ satisfying $\xi \gamma=0$. Also if $\gamma=0$ then a q.S. manifold is a cosymplectic manifold [10], the converse is true. From (2.1), we have [9]

$$
\left(\nabla_{U} \varphi\right) V=\gamma\{g(U, V) \xi-\eta(U) V\}
$$

Again from (2.1) and (2.2), we get

$$
R(U, V) \xi=-U[\gamma] \varphi V+V[\gamma] \varphi U+\gamma^{2}\{\eta(V) U-\eta(U) V\}
$$

Using (2.1) and (2.2), we obtain

$$
R(U, \xi) \xi=\gamma^{2}\{U-\eta(U) \xi\}
$$

and

$$
R(U, \xi) V=-U[\gamma] \varphi V-\gamma^{2}\{g(U, V) \xi-\eta(V) U\} .
$$

In a three-dimensional Riemannian manifold, the curvature tensor is written

$$
R(U, V) W=g(V, W) Q U-g(U, W) Q V+S(V, W) U-S(U, W) V-\frac{r}{2}\{g(V, W) U-g(U, W) V\} .
$$

Let $M$ be a three-dimensional q.S. manifold. The Ricci tensor $S$ of $M$ is

$$
S(V, W)=\left(\frac{r}{2}-\gamma^{2}\right) g(V, W)+\left(3 \gamma^{2}-\frac{r}{2}\right) \eta(V) \eta(W)-\eta(V) d \gamma(\varphi W)-\eta(W) d \gamma(\varphi V)
$$

From (2.3), we get

$$
Q V=\left(\frac{r}{2}-\gamma^{2}\right) V+\left(3 \gamma^{2}-\frac{r}{2}\right) \eta(V) \xi+\eta(V)(\varphi \operatorname{grad} \gamma)-d \gamma(\varphi V) \xi
$$

where $d \gamma(V)=g(\operatorname{grad} \gamma, V)$. Again from (2.3), we obtain

$$
S(U, \xi)=2 \gamma^{2} \eta(U)-d \gamma(\varphi U) .
$$

As a consequence of (2.1), we have

$$
\left(\nabla_{U} \eta\right) W=g\left(\nabla_{U} \xi, W\right)=-\gamma g(\varphi U, V)
$$

\section{Three-dimensional q.S. manifolds admitting S.K.con.}

For an a.c.m. manifold $M$, the S.K.con. $\widetilde{\nabla}$ is given by [4]

$$
\widetilde{\nabla}_{U} V=\nabla_{U} V-\eta(V) \nabla_{U} \xi+\left(\nabla_{U} \eta\right)(V) \xi .
$$

Let $M^{3}$ be a q.S. manifold. Then using (3.1), we have

$$
\widetilde{\nabla}_{U} V=\nabla_{U} V+\gamma \eta(V) \varphi U+\gamma g(U, \varphi V) \xi .
$$

Now we put equation (3.1) in the definition of the Riemannian curvature tensor, we can write

$$
\widetilde{R}(U, V) W=\widetilde{\nabla}_{U} \widetilde{\nabla}_{V} W-\widetilde{\nabla}_{V} \widetilde{\nabla}_{U} W-\widetilde{\nabla}_{[U, V]} W .
$$

Using (3.2) in (3.3), we obtain

$$
\begin{aligned}
\widetilde{R}(U, V) W= & \widetilde{\nabla}_{U}\left(\nabla_{V} W+\gamma \eta(W) \varphi V+\gamma g(V, \varphi W) \xi\right) \\
& -\widetilde{\nabla}_{V}\left(\nabla_{U} W+\gamma \eta(W) \varphi U+\gamma g(U, \varphi W) \xi\right) \\
& -\left(\nabla_{[U, V]} W+\gamma \eta(W) \varphi[U, V]+\gamma g([U, V], \varphi W) \xi .\right.
\end{aligned}
$$

Again using (2.2) and (2.4) in (3.4), we have

$$
\begin{aligned}
\widetilde{R}(U, V) W= & R(U, V) W+U[\gamma]\{g(V, \varphi W) \xi+\eta(W) \varphi V\} \\
& -V[\gamma]\{g(U, \varphi W) \xi+\eta(W) \varphi U\} \\
& +\gamma^{2}\{g(U, W) \eta(V) \xi-g(V, W) \eta(U) \xi+\eta(U) \eta(W) V \\
& -\eta(V) \eta(W) U+g(U, \varphi W) \varphi V-g(V, \varphi W) \varphi U\}
\end{aligned}
$$

which gives

$$
\begin{aligned}
g(\widetilde{R}(U, V) W, Z)= & g(R(U, V) W, Z) \\
& +U[\gamma]\{g(V, \varphi W) \eta(Z)+g(\varphi V, Z) \eta(W)\} \\
& -Y[\gamma]\{g(U, \varphi W) \eta(Z)+g(\varphi U, Z) \eta(W)\} \\
& +\gamma^{2}\{g(U, W) \eta(V) \eta(Z)-g(V, W) \eta(U) \eta(Z) \\
& +g(V, Z) \eta(U) \eta(W)-g(U, Z) \eta(V) \eta(W) \\
& +g(U, \varphi W) g(\varphi V, Z)-g(V, \varphi W) g(\varphi U, Z)\} .
\end{aligned}
$$


Putting $U=Z=e_{i},\{i=1,2,3\}$, in (3.6), we get

$$
\widetilde{S}(V, W)=S(V, W)+(\varphi V)[\gamma] \eta(W)-2 \gamma^{2} \eta(V) \eta(W) .
$$

From (3.7), we have

$$
\widetilde{Q} V=Q V+(\varphi V)[\gamma] \xi-2 \gamma^{2} \eta(V) \xi
$$

Again putting $V=W=e_{i}$ in (3.7), then we obtain

$$
\widetilde{r}=r-2 \gamma^{2}
$$

From (3.5) and (3.6), we have

$$
\begin{gathered}
\widetilde{R}(U, V) W+\widetilde{R}(V, U) W=0, \\
g(\widetilde{R}(U, V) W, Z)+g(\widetilde{R}(U, V) Z, W)=0 .
\end{gathered}
$$

and

$$
\begin{aligned}
\widetilde{R}(U, V) W+\widetilde{R}(V, W) U+\widetilde{R}(W, U) V= & U[\gamma]\{2 g(V, \varphi W) \xi+\eta(W) \varphi V-\eta(V) \varphi W\} \\
& +V[\gamma]\{2 g(W, \varphi U) \xi+\eta(U) \varphi W-\eta(W) \varphi U\} \\
& +W[\gamma]\{2 g(U, \varphi V) \xi+\eta(V) \varphi U-\eta(U) \varphi V\}
\end{aligned}
$$

If $\gamma$ is a constant, then we have

$$
\widetilde{R}(U, V) W+\widetilde{R}(V, W) U+\widetilde{R}(W, U) V=0
$$

\section{Three-dimensional q.S. manifolds and $\mathscr{D}_{\alpha}$-homothetic deformations}

In this section, we study a $\mathscr{D}_{\alpha}$-homothetic deformation on a q.S. manifold $M^{3}$.

For a $(2 n+1)$-dimensional a.c.m. manifold $(M, \varphi, \xi, \eta, g)$ if $\eta=0$, then there is an $2 n$-dimensional distribution $\mathscr{D} \alpha$ on $M$. Also an $2 n$-dimensional homothetic deformation or a $\mathscr{D}_{\alpha}$-homothetic deformation is defined by

$$
\begin{aligned}
\eta^{\alpha} & =\alpha \eta, \quad \xi^{\alpha}=\frac{1}{\alpha} \xi, \quad \varphi^{\alpha}=\varphi, \\
g^{\alpha} & =\alpha g+\alpha(\alpha-1) \eta \otimes \eta,
\end{aligned}
$$

where $\alpha=$ constant $>0$. If $(M, \varphi, \xi, \eta, g)$ is an a.c.m. structure then $\left(M, \varphi^{\alpha}, \xi^{\alpha}, \eta^{\alpha}, g^{\alpha}\right)$ is also an a.c.m. structure [2]. Now we have the followings:

Lemma 4.1. Let $M^{3}$ be a q.S. manifold admitting a $\mathscr{D}_{\alpha}$-homothetic deformation. Then

$$
\nabla_{U}^{\alpha} V=\nabla_{U} V-(\alpha-1) \gamma\{\eta(U) \varphi V+\eta(V) \varphi U\} .
$$

Proof. From Kozsul's formula, we have

$$
2 g^{\alpha}\left(\nabla_{U}^{\alpha} V, W\right)=U g^{\alpha}(V, W)+V g^{\alpha}(U, W)-W g^{\alpha}(U, V)-g^{\alpha}(U,[V, W])-g^{\alpha}(V,[U, W])+g^{\alpha}(W,[U, V]),
$$

for any vector fields $U, V, W$. From (4.1), we obtain

$$
\begin{aligned}
2\left\{\alpha g\left(\nabla_{U}^{\alpha} V, W\right)+\alpha(\alpha-1) \eta\left(\nabla_{U}^{\alpha} V\right) \eta(W)\right\}= & U\{\alpha g(V, W)+\alpha(\alpha-1) \eta(U) \eta(W)\} \\
& +V\{\alpha g(U, W)+\alpha(\alpha-1) \eta(U) \eta(W)\} \\
& -W\{\alpha g(U, V)+\alpha(\alpha-1) \eta(U) \eta(V)\} \\
& -\alpha\{g(U,[V, W])+(\alpha-1) \eta(U) \eta([V, W])\} \\
& -\alpha\{g(V,[U, W])+(\alpha-1) \eta(V) \eta([U, W])\} \\
& +\alpha\{g(W,[U, V])+(\alpha-1) \eta(W) \eta([U, V])\} .
\end{aligned}
$$

After some calculations, we get

$$
\begin{aligned}
2\left\{\alpha g\left(\nabla_{U}^{\alpha} V, W\right)+\alpha(\alpha-1) \eta\left(\nabla_{U}^{\alpha} V\right) \eta(W)\right\}= & \alpha\left\{g\left(\nabla_{U} V, W\right)+g\left(V, \nabla_{U} W\right)\right\} \\
& +\alpha(\alpha-1)\{U(\eta(V) \eta(W))+V(\eta(U) \eta(W)) \\
& -W(\eta(U) \eta(V))-\eta(U) \eta\left(\nabla_{V} W\right)+\eta(U) \eta\left(\nabla_{W} V\right) \\
& -\eta(V) \eta\left(\nabla_{U} W\right)+\eta(V) \eta\left(\nabla_{W} U\right) \\
& \left.+\eta(W) \eta\left(\nabla_{U} V\right)-\eta(W) \eta\left(\nabla_{V} U\right)\right\} .
\end{aligned}
$$

Thus we have

$$
\begin{aligned}
2\left\{\alpha g\left(\nabla_{U}^{\alpha} V, W\right)+\alpha(\alpha-1) \eta\left(\nabla_{U}^{\alpha} V\right) \eta(W)\right\}= & 2 \alpha g\left(\nabla_{U} V, W\right)+2 a(a-1) \eta\left(\nabla_{U} V\right) \eta(W) \\
& +a(a-1)\left\{g\left(V, \nabla_{U} \xi\right) \eta(W)+g\left(W, \nabla_{U} \xi\right) \eta(V)\right. \\
& +g\left(U, \nabla_{V} \xi\right) \eta(W)+g\left(W, \nabla_{V} \xi\right) \eta(U) \\
& \left.-g\left(U, \nabla_{W} \xi\right) \eta(V)-g\left(V, \nabla_{W} \xi\right) \eta(U)\right\} .
\end{aligned}
$$


Using (2.1) in (4.3), we get

$$
\begin{aligned}
2\left\{\alpha g\left(\nabla_{U}^{\alpha} V, W\right)+\alpha(\alpha-1) \eta\left(\nabla_{U}^{\alpha} V\right) \eta(W)\right\}= & 2 \alpha g\left(\nabla_{U} V, W\right)+2 a(a-1) \eta\left(\nabla_{U} V\right) \eta(W) \\
& -\alpha(\alpha-1) \gamma\{g(V, \varphi U) \eta(W)+g(W, \varphi U) \eta(V) \\
& +g(\varphi V, U) \eta(W)+g(W, \varphi V) \eta(U) \\
& -g(U, \varphi W) \eta(V)-g(V, \varphi W) \eta(U)\} .
\end{aligned}
$$

After some calculations, we obtain

$$
g\left(\nabla_{U}^{\alpha} V, W\right)+(\alpha-1) \eta\left(\nabla_{U}^{\alpha} V\right) \eta(W)=g\left(\nabla_{U} V, W\right)+(\alpha-1) \eta\left(\nabla_{U} V\right) \eta(W)-(\alpha-1) \gamma\{g(W, \varphi U) \eta(V)+g(W, \varphi V) \eta(U)\},
$$

which implies (4.2).

Proposition 4.2. Let $M^{3}$ be a q.S. manifold with a $\mathscr{D}_{a}$-homothetic deformation. Then

$$
\begin{aligned}
R^{\alpha}(U, V) W= & R(U, V) W \\
& -(\alpha-1) U[\gamma]\{\eta(V) \varphi W+\eta(W) \varphi V\} \\
& +(\alpha-1) V[\gamma]\{\eta(U) \varphi W+\eta(W) \varphi U\} \\
& -(\alpha-1) \gamma^{2}\{\eta(U) \eta(W) V-\eta(V) \eta(W) U\} \\
& +(\alpha-1) \gamma^{2}\{g(V, W) \eta(U) \xi-g(U, W) \eta(V) \xi \\
& -2 \eta(U) \eta(W) V+2 \eta(V) \eta(W) U-2 g(U, \varphi V) \varphi W \\
& -g(U, \varphi W) \varphi V+g(V, \varphi W) \varphi U\} .
\end{aligned}
$$

Proof. The definition of the Riemannian curvature tensor, we can write

$$
R^{\alpha}(U, V) W=\nabla_{U}^{\alpha} \nabla_{V}^{\alpha} W-\nabla_{V}^{\alpha} \nabla_{U}^{\alpha} W-\nabla_{[U, V]}^{\alpha} W .
$$

Using (4.2) in (4.5) and after long calculations, we have

$$
\begin{aligned}
R^{\alpha}(U, V) W= & R(U, V) W \\
& -(\alpha-1) \gamma\left[\eta(U) \varphi \nabla_{V} W-\eta(U) \nabla_{V} \varphi W-\eta(V) \varphi \nabla_{U} W\right. \\
& +\eta(V) \nabla_{U} \varphi W-\eta(W) \varphi \nabla_{U} V+\eta(W) \nabla_{U} \varphi V \\
& +\eta(W) \varphi \nabla_{V} U-\eta(W) \nabla_{V} \varphi U \\
& +a \gamma\{2 g(U, \varphi V) \varphi W+g(U, \varphi W) \varphi V-g(V, \varphi W) \varphi U\} \\
& +(\alpha-1) \gamma\{\eta(U) \eta(W) V-\eta(V) \eta(W) U\}] \\
& -(\alpha-1)\{(U(\gamma))(\eta(V) \varphi W+\eta(W) \varphi V) \\
& -(V(\gamma))(\eta(U) \varphi W+\eta(W) \varphi U)\} .
\end{aligned}
$$

Using (2.2) in (4.6), we obtain (4.4).

From (4.4), we have

$$
R^{\alpha}(U, V) W+R^{\alpha}(V, U) W=0,
$$

and

$$
R^{\alpha}(U, V) W+R^{\alpha}(V, W) U+R^{\alpha}(W, U) V=0 .
$$

\section{5. $\mathscr{D}_{\alpha}$-homothetic deformations on three-dimensional q.S. manifolds admitting the S.K.con.}

In this section, we study how a $\mathscr{D}_{\alpha}$-homothetic deformation affects a three-dimensional q.S. manifold $M$ admitting the S.K.con..

Lemma 5.1. Let $M^{3}$ be a q.S. manifold with a $\mathscr{D}_{\alpha}$-homothetic deformation admitting the S.K.con.. Then

$$
\widetilde{\nabla}_{U}^{\alpha} V=\nabla_{U} V-(\alpha-1) \gamma \eta(U) \varphi V+\gamma \eta(V) \varphi U+\gamma g(U, \varphi V) \xi
$$

Proof. Using (3.1) and (4.2), we obtain

$$
\begin{aligned}
\widetilde{\nabla}_{U}^{\alpha} V= & \nabla_{U} V-\gamma \eta^{\alpha}(V) \varphi^{\alpha} U+\gamma g^{\alpha}(U, \varphi V) \xi^{\alpha} \\
= & \nabla_{U} V-(\alpha-1) \gamma\{\eta(U) \varphi V+\eta(V) \varphi U\}-\gamma \alpha \eta(V) \varphi U \\
& +\gamma[g(U, \varphi V)+(\alpha-1) \eta(U) \eta(\varphi V)] \xi \\
= & \nabla_{U} V-(\alpha-1) \gamma \eta(U) \varphi V+\gamma \eta(V) \varphi U+\gamma g(U, \varphi V) \xi
\end{aligned}
$$


Proposition 5.2. Let $M^{3}$ be a q.S. manifold with a $\mathscr{D} \alpha$-homothetic deformation admitting the S.K.con.. Then

$$
\begin{aligned}
\widetilde{R}^{\alpha}(U, V) W= & R(U, V) W \\
& +U[\gamma]\{g(V, \varphi W) \xi+\eta(W) \varphi V-(\alpha-1) \eta(V) \varphi W\} \\
& -V[\gamma]\{g(U, \varphi W) \xi+\eta(W) \varphi U-(\alpha-1) \eta(U) \varphi W\} \\
& +\gamma^{2}\{g(U, W) \eta(V) \xi-g(V, W) \eta(U) \xi+g(U, \varphi W) \varphi V \\
& -g(V, \varphi W) \varphi U+\eta(U) \eta(W) V-\eta(V) \eta(W) U\} \\
& -2(\alpha-1) \gamma^{2} g(U, \varphi V) \varphi W .
\end{aligned}
$$

Proof. Using (4.1), (3.5) and (4.4), we have

$$
\begin{aligned}
\widetilde{R}^{\alpha}(U, V) W= & R^{\alpha}(U, V) W+U[\gamma]\left\{g^{\alpha}\left(V, \varphi^{\alpha} W\right) \xi^{\alpha}+\eta^{\alpha}(W) \varphi^{\alpha} V\right\} \\
& -V[\gamma]\left\{g^{\alpha}\left(U, \varphi^{\alpha} W\right) \xi^{\alpha}+\eta^{\alpha}(W) \varphi^{\alpha} U\right\} \\
& +\gamma^{2}\left\{g^{\alpha}(U, W) \eta^{\alpha}(V) \xi^{\alpha}-g^{\alpha}(V, W) \eta^{\alpha}(U) \xi^{\alpha}\right. \\
& +\eta^{\alpha}(U) \eta^{\alpha}(W) V-\eta^{\alpha}(V) \eta^{\alpha}(W) U \\
& \left.+g^{\alpha}\left(U, \varphi^{\alpha} W\right) \varphi^{\alpha} V-g^{\alpha}\left(V, \varphi^{\alpha} W\right) \varphi^{\alpha} U\right\}
\end{aligned}
$$

Using (4.2) in (5.3), we obtain (5.2).

Now taking the inner product with $Z$ and putting $V=W=e_{i},\{i=1,2,3\}$, in (5.2), we get

$$
\widetilde{S}^{\alpha}(U, Z)=S(U, Z)+(\varphi U)[\gamma] \eta(Z)+(\alpha-1)(\varphi Z)[\gamma] \eta(U)-2 \gamma^{2} \eta(U) \eta(Z)
$$

If we use (2.3) in (5.4), we have

$$
\begin{aligned}
\tilde{S}^{\alpha}(U, Z)= & \left(\frac{r}{2}-\gamma^{2}\right) g(U, Z)+\left(3 \gamma^{2}-\frac{r}{2}\right) \eta(U) \eta(Z) \\
& -(\varphi U)[\gamma] \eta(Z)-(\varphi Z)[\gamma] \eta(U) \\
& +(\varphi U)[\gamma] \eta(Z)+(\alpha-1)(\varphi Z)[\gamma] \eta(U) \\
& -2 \gamma^{2} \eta(U) \eta(Z)
\end{aligned}
$$

i.e.,

$$
\tilde{S}^{\alpha}(U, Z)=\left(\frac{r}{2}-\gamma^{2}\right)\{g(U, Z)-\eta(U) \eta(Z)\}+(\alpha-2)(\varphi Z)[\gamma] \eta(U) .
$$

Also we take $U=Z=e_{i}$ in (5.4), we get

$$
\widetilde{r}^{\alpha}=r-2 \gamma^{2}
$$

\section{Main result}

In this section, we study a projectively flat q.S. manifold $M^{3}$ with a $\mathscr{D}_{\alpha}$-homothetic deformation admitting the S.K.con..

In a q.S. manifold $M^{3}$ with a $\mathscr{D}_{\alpha}$-homothetic deformation admitting the S.K.con. $\widetilde{\nabla}^{a}$, the projective curvature tensor $\widetilde{P}^{a}$ is given by

$$
\widetilde{P}^{\alpha}(U, V) W=\widetilde{R}^{\alpha}(U, V) W-\frac{1}{2}\left\{\widetilde{S}^{\alpha}(V, W) U-\widetilde{S}^{\alpha}(U, W) V\right\} .
$$

Now let $M^{3}$ be a projectively flat q.S. manifold with a $\mathscr{D} \alpha$-homothetic deformation admitting the S.K.con. $\widetilde{\nabla}^{a}\left(\right.$ i.e. $\left.\widetilde{P}^{a}=0\right)$. Then we have

$$
\widetilde{R}^{\alpha}(U, V) W=\frac{1}{2}\left\{\widetilde{S}^{\alpha}(V, W) U-\widetilde{S}^{\alpha}(U, W) V\right\}
$$

Using (5.2) and (5.4) in (6.1), we get

$$
\begin{aligned}
& R(U, V) W \\
& +U[\gamma]\{g(V, \varphi W) \xi+\eta(W) \varphi V-(\alpha-1) \eta(V) \varphi W\} \\
& -V[\gamma]\{g(U, \varphi W) \xi+\eta(W) \varphi U-(\alpha-1) \eta(U) \varphi W\} \\
& +\gamma^{2}\{g(U, W) \eta(V) \xi-g(V, W) \eta(U) \xi+g(U, \varphi W) \varphi V \\
& -g(V, \varphi W) \varphi U+\eta(U) \eta(W) V-\eta(V) \eta(W) U\} \\
& +2(\alpha-1) \gamma^{2} g(U, \varphi V) \varphi W \\
& =\quad \frac{1}{2}[S(V, W) U+(\varphi V)[\gamma] \eta(W) U+(\alpha-1)(\varphi W)[\gamma] \eta(V) U \\
& -2 \gamma^{2} \eta(V) \eta(W) U-S(U, W) V-(\varphi U)[\gamma] \eta(W) V \\
& \left.-(\alpha-1)(\varphi W)[\gamma] \eta(U) V+2 \gamma^{2} \eta(U) \eta(W) W\right],
\end{aligned}
$$


which gives

$$
\begin{aligned}
& g(R(U, V) W, Z) \\
+ & +U[\gamma]\{g(V, \varphi W) \eta(Z)+\eta(W) g(\varphi V, Z)-(\alpha-1) \eta(V) g(\varphi W, Z)\} \\
& -V[\gamma]\{g(U, \varphi W) \eta(Z)+\eta(W) g(\varphi U, Z)-(\alpha-1) \eta(U) g(\varphi W, Z)\} \\
+ & \gamma^{2}\{g(U, W) \eta(V) \eta(Z)-g(V, W) \eta(U) \eta(Z)+g(U, \varphi W) g(\varphi V, Z) \\
& -g(V, \varphi W) g(\varphi U, Z)+\eta(U) \eta(W) g(V, Z)-\eta(V) \eta(W) g(U, Z)\} \\
& +2(\alpha-1) \gamma^{2} g(U, \varphi V) g(\varphi W, Z) \\
= & \frac{1}{2}[S(V, W) g(U, Z)+(\varphi V)[\gamma] \eta(W) g(U, Z) \\
& +(\alpha-1)(\varphi W)[\gamma] \eta(V) g(U, Z)-2 \gamma^{2} \eta(V) \eta(W) g(U, Z) \\
& -S(U, W) g(V, Z)-(\varphi U)[\gamma] \eta(W) g(V, Z) \\
& \left.-(\alpha-1)(\varphi W)[\gamma] \eta(U) g(V, Z)+2 \gamma^{2} \eta(U) \eta(W) g(V, Z)\right] .
\end{aligned}
$$

Putting $U=Z=\xi$ in (6.2), we have

$$
S(V, W)=2 \gamma^{2} \eta(V) \eta(W)-(\varphi W)[\gamma] \eta(V)-(\varphi V)[\gamma] \eta(W) .
$$

If we put (6.3) in (5.4), we get

$$
\widetilde{S}^{\alpha}(V, W)=(\alpha-2)(\varphi W)[\gamma] \eta(V) .
$$

Clearly, if $\gamma$ is a constant or $\alpha=2$, then from (6.4), we have $\widetilde{S}^{\alpha}=0$. If $\widetilde{S}^{\alpha}=0$, then from (6.1), we get $\widetilde{R}^{\alpha}=0$. Conversely if $\widetilde{R}^{\alpha}=0$ then we have $\widetilde{S}^{\alpha}=0$ and from (6.4) we obtain $\gamma$ is a constant or $\alpha=2$.

Thus the above discussion leads us to state the following:

Theorem 6.1. Let $M^{3}$ be a projectively flat q.S. manifold with a $\mathscr{D}_{\alpha}$-homothetic deformation admitting the S.K.con.. Then the followings hold: (i) $\gamma$ is a constant or $\alpha=2$. (ii) The manifold $M$ is a Ricci-flat manifold, (iii) The manifold $M$ is a flat manifold.

\section{Example}

In this section, we give an example of three-dimensional q.S. manifolds with a $\mathscr{D}_{\alpha}$-homothetic deformation admitting the S.K.con.. Let $M=\left\{(x, y, z) \in \mathfrak{R}^{3}: x \neq 0\right\}$ be a three-dimensional manifold, where $(x, y, z)$ are standard coordinates in $\mathfrak{R}^{3}$ and $\left\{\tilde{e}_{1}, \tilde{e}_{2}, \tilde{e}_{3}\right\}$ be linearly independent global frame on $M$ is given by

$$
\tilde{e}_{1}=2 \frac{\partial}{\partial y}, \quad \tilde{e}_{2}=2 \frac{\partial}{\partial x}-4 \frac{\partial}{\partial y}+y \frac{\partial}{\partial z}, \quad \tilde{e}_{3}=\frac{\partial}{\partial z} .
$$

Let $g$ be the Riemannian metric, $\eta$ be the 1-form and $\varphi$ be the $(1,1)$-type tensor field given by

$$
\begin{gathered}
g\left(\tilde{e}_{1}, \tilde{e}_{3}\right)=g\left(\tilde{e}_{1}, \tilde{e}_{2}\right)=g\left(\tilde{e}_{2}, \tilde{e}_{3}\right)=0, \\
g\left(\tilde{e}_{1}, e_{1}\right)=g\left(\tilde{e}_{2}, \tilde{e}_{2}\right)=g\left(\tilde{e}_{3}, \tilde{e}_{3}\right)=1, \\
\eta(W)=g\left(W, \tilde{e}_{3}\right), \\
\varphi \tilde{e}_{1}=\tilde{e}_{2}, \quad \varphi \tilde{e}_{2}=-\tilde{e}_{1}, \quad \varphi \tilde{e}_{3}=0,
\end{gathered}
$$

respectively. Using the linearity of $\varphi$ and $g$, we have

$$
\eta\left(\tilde{e}_{3}\right)=1, \quad \varphi^{2} W=-W+\eta(W) \tilde{e}_{3},
$$

and

$$
g(\varphi W, \varphi Z)=g(W, Z)-\eta(W) \eta(Z) .
$$

Thus for $\tilde{e}_{3}=\xi,(\varphi, \xi, \eta, g)$ defines a c.m.s. on $M^{3}$. Thus we have

$$
\left[\tilde{e}_{1}, \tilde{e}_{2}\right]=2 \tilde{e}_{3}, \quad\left[\tilde{e}_{1}, \tilde{e}_{3}\right]=0, \quad\left[\tilde{e}_{2}, \tilde{e}_{3}\right]=0 .
$$

Recall Koszul's formula

$$
2 g\left(\nabla_{U} V, W\right)=U g(V, W)+V g(U, W)-W g(U, V)-g(U,[V, W])-g(V,[U, W])+g(W,[U, V]),
$$

Taking $\tilde{e}_{3}=\xi$ and using the above formula for Riemannian metric $g$, we get

$$
\begin{array}{llll}
\nabla_{\tilde{e}_{1}} \tilde{e}_{3} & =-\tilde{e}_{2}, & \nabla_{\tilde{e}_{2}} \tilde{e}_{3}=\tilde{e}_{1}, & \nabla_{\tilde{e}_{3} \tilde{e}_{3}}=0, \\
\nabla_{\tilde{e}_{3}} \tilde{e}_{1}=-\tilde{e}_{2}, & \nabla_{\tilde{e}_{1}} \tilde{e}_{2}=\tilde{e}_{3}, & \nabla_{\tilde{e}_{2}} \tilde{e}_{1}=-\tilde{e}_{3}, \\
\nabla_{\tilde{e}_{2}} \tilde{e}_{2}=0, & \nabla_{\tilde{e}_{3}} \tilde{e}_{2}=\tilde{e}_{1}, & \nabla_{\tilde{e}_{1}} \tilde{e}_{1}=0 .
\end{array}
$$


Hence from (2.1), the manifold $M^{3}$ is a q.S. manifold with $\gamma=1$. Using (7.1), (7.2), (7.3) and (7.4) in (5.1), we have $D_{a}$-homothetic deformation of the manifold $M^{3}$ admitting the S.K.con. given by

$$
\begin{array}{llll}
\widetilde{\nabla}_{\tilde{e}_{1}}^{\alpha} \tilde{e}_{3}=0, & \widetilde{\nabla}_{\tilde{e}_{2}}^{\alpha} \tilde{e}_{3}=0, & \widetilde{\nabla}_{e_{3}}^{\alpha} \tilde{e}_{3}=0, \\
\widetilde{\nabla}_{\tilde{e}_{3}}^{\alpha} \tilde{e}_{1}=-\alpha \tilde{e}_{2}, & \widetilde{\nabla}_{\tilde{e}_{1}}^{\alpha} \tilde{e}_{2}=0, & \widetilde{\nabla}_{\tilde{e}_{2}}^{\alpha} \tilde{e}_{1}=0, \\
\widetilde{\nabla}_{\tilde{e}_{2}}^{\alpha} \tilde{e}_{2}=0, & \widetilde{\nabla}_{\tilde{e}_{3}}^{\alpha} \tilde{e}_{2}=\alpha e_{1}, & \widetilde{\nabla}_{\tilde{e}_{1}}^{\alpha} \tilde{e}_{1}=0 .
\end{array}
$$

Using (7.5), we obtain

$$
\begin{array}{rlrlrl}
\widetilde{R}^{\alpha}\left(\tilde{e}_{1}, \tilde{e}_{2}\right) \tilde{e}_{1} & =2 \alpha \tilde{e}_{2}, & \widetilde{R}^{\alpha}\left(\tilde{e}_{1}, \tilde{e}_{2}\right) \tilde{e}_{2}=-2 \alpha \tilde{e}_{2}, & & \widetilde{R}^{\alpha}\left(\tilde{e}_{1}, \tilde{e}_{2}\right) \tilde{e}_{3}=0, \\
\widetilde{R}^{\alpha}\left(\tilde{e}_{1}, \tilde{e}_{3}\right) \tilde{e}_{1}=0, & \widetilde{R}^{\alpha}\left(\tilde{e}_{1}, \tilde{e}_{3}\right) \tilde{e}_{2}=0, & & \widetilde{R}^{\alpha}\left(\tilde{e}_{1}, \tilde{e}_{3}\right) \tilde{e}_{3}=0, \\
\widetilde{R}^{\alpha}\left(\tilde{e}_{2}, \tilde{e}_{3}\right) \tilde{e}_{1}=0, & \widetilde{R}^{\alpha}\left(\tilde{e}_{2}, \tilde{e}_{3}\right) \tilde{e}_{2}=0, & & \widetilde{R}^{\alpha}\left(\tilde{e}_{2}, \tilde{e}_{3}\right) \tilde{e}_{3}=0 .
\end{array}
$$

Thus from (7.6), the manifold $M^{3}$ is a flat manifold. Since a flat manifold is a Ricci flat manifold, from the Theorem 6.1 the manifold $M^{3}$ is a projectively flat manifold.

\section{References}

[1] D. E. Blair, The theory of quasi-Sasakian structure, J. Differential Geo., 1(3-4) (1967), 331-345.

[2] S. Tanno, Quasi-Sasakian structure of rank 2p+1, J. Differential Geom., 5(3-4) (1971), 317-324.

[3] Z. Olszak, On three dimensional conformally flat quasi-Sasakian manifolds, Period Math. Hungar., 33(2) (1996), 105-113.

[4] Z. Olszak, The Schouten-van Kampen affine connection adapted an almost (para) contact metric structure, Publ. De L'inst. Math., 94(108) (2013), $31-42$.

[5] A. Yildız, f-Kenmotsu manifolds with the Schouten-van Kampen connection, Publ. De L'Inst. Math., 102(116) (2017), 93-105.

[6] D. E. Blair, Contact Manifolds in Riemannian Geometry, Lecture Notes in Mathematics Vol. 509, Springer-Verlag, Berlin-New York, 1976

[7] D. E. Blair, Riemannian Geometry of Contact and Symplectic Manifolds, Progress in Mathematics, Vol. 203, Birkhäuser, Boston, 2002.

[8] K. Yano, M. Kon, Structures on Manifolds, World Scientific, 1984

[9] Z. Olszak, Normal almost contact metric manifolds of dimension 3, Ann. Polon. Math., 1(47) (1986), 41-50.

[10] D. Janssens, L. Vanhecke, Almost contact structures and curvature tensors, Kodai Math. J., 4(1) (1981), 1-27. 